\title{
NONNEGATIVE REFLEXIVE GENERALIZED INVERSES AND APPLICATIONS TO GROUP MONOTONICITY
}

\author{
SACHINDRANATH JAYARAMAN
}

\begin{abstract}
A classical finite dimensional result of Berman and Plemmons says that a nonnegative matrix with a nonnegative reflexive generalized inverse has a nonnegative rank factorization. In this article, we propose a notion of nonnegative rank factorization that is applicable in the infinite dimensional setting over more general cones and prove an infinite dimensional generalization of Berman and Plemmons's result. As a consequence, a simpler proof of the finite dimensional result (on the existence of nonnegative rank factorizations) is obtained. Characterizations of nonnegativity of the group inverse (when it exists) in infinite dimensions are also presented.
\end{abstract}

Mathematics subject classification (2010): 47B60, 47B65, 15A09.

Keywords and phrases: nonnegative reflexive generalized inverse, nonnegative rank factorization, selfdual cones, nonnegative group inverse.

\section{REFERENCES}

[1] A. Ben-IsRael And T. N. E. GReville, Generalized Inverses: Theory and Applications, $2^{\text {nd }}$ ed., Springer Verlag, New York, 2003.

[2] A. Berman and R. J. Plemmons, Rank factorizations of nonnegative matrices: Problems and Solutions, $73-14$ (Question), SIAM Rev., 15, 3, July (1973), 655.

[3] A. Berman ANd R. J. Plemmons, Inverses of nonnegative matrices, Linear Multilinear Algebra, 2 (1974), 161-172.

[4] A. Berman and R. J. Plemmons, Matrix group monotonicity, Proc. Amer. Math. Soc., 46 (1974), 355-359.

[5] A. Berman and R. J. Plemmons, Nonnegative Matrices in the Mathematical Sciences, $2^{\text {nd }}$ ed., SIAM, Philadelphia, 1994.

[6] S. L. Campbell and G. D. Poole, Computing nonnegative rank factorizations, Linear Algebra Appl., 35 (1981), 175-182.

[7] S. R. CARAdus, Generalized inverses and operator theory, Queen's Papers in Pure and Applied Mathematics, Queen's University, Kingston, 1978.

[8] A. Cegiels KI, Obtuse cones and Gram matrices with nonnegative inverse, Linear Algebra Appl., 335 (2001), 167-181.

[9] J.-C. CHEN, The nonnegative rank factorization of nonnegative matrices, Linear Algebra Appl., 62 (1984), 207-217.

[10] L. Collatz, Functional Analysis and Numerical Mathematics, Academic Press, New York, 1966.

[11] E. DEUTSCH, Semiinverses, reflexive semiinverses and pseudoinverses of an arbitrary linear transformation, Linear Algebra Appl., 4 (1971), 313-322.

[12] P. FLoR, On groups of nonnegative matrices, Compositio Math., 21 (1969), 376-382.

[13] J. L. GoffIN, The relaxation method for solving systems of linear inequalities, Math. Oper. Res., 5, 3 (1980), 388-414.

[14] S. K. JAIN AND J. TYNAN, Nonnegative rank factorization of a nonnegative matrix $A$ with $A^{\dagger} A$ nonnegative, Linear Multilinear Algebra, 51, 1 (2003), 83-95.

[15] S. K. JAIn AND J. TYNAN, Nonnegative matrices A with AA nonnegative, Linear Algebra Appl., 379 (2004), 381-394. 
[16] Sachindranath Jayaraman And K. C. Sivakumar, Weak monotonicity of Hilbert space operators, Int. J. Pure Appl. Math., 29, 1 (2006), 65-79.

[17] Sachindranath Jayaraman And K. C. Sivakumar, Weak monotonicity of a versus $\{1\}$ inverses nonnegative on the range space of A, Linear Algebra Appl., 427, 2-3 (2007), 171-175.

[18] M. W. JeTER AND W. C. PyE, A note on nonnegative rank factorizations, Linear Algebra Appl., 38 (1981), 171-173.

[19] M. W. JETER AND W. C. PYE, Some nonnegative matrices without nonnegtive rank factorization, Indust. Math., 32, 1 (1982), 37-41.

[20] M. W. JETER AND W. C. PYE, Some duality theorems for nonnegative rank factorizations, Indust. Math., 33, 1 (1983), 63-71.

[21] S. H. KUlKaRni AND K. C. SivaKumar, Three types of operator monotonicity, J. Anal., 12 (2004), $153-163$.

[22] T. Kurmayya And K. C. Sivakumar, Nonnegative Moore-Penrose inverses of Gram operators, Linear Algebra Appl., 422 (2007), 471-476.

[23] T. Kurmayya AND K. C. SivaKumar, Nonnegative Moore-Penrose inverses of operators over Hilbert spaces, Positivity, 12, 3 (2008), 475-481.

[24] A. NoviKoff, A characterization of operators mapping a cone into its dual, Proc. Amer. Math. Soc., 16 (1965), 356-359.

[25] J. E. Peris AND B. SubiZA, A characterization of weak monotone matrices, Linear Algebra Appl., 166 (1992), 167-184.

[26] R. PiziaK AND P. L. Odell, Full rank factorization of matrices, Math. Mag., 72, 3 (1999), 193-201.

[27] D. J. Richman AND H. SCHNEIDER, Primes in the semigroup of nonnegative matrices, Linear Multilinear Algebra, 2 (1974), 135-140.

[28] P. ROBERT, On the group inverse of a linear transformation, J. Math. Anal. Appl., 22 (1968), 658-669.

[29] K. C. Siva Kumar, Nonnegative generalized inverses, Indian J. Pure Appl. Math., 28, 7 (1997), 939942.

[30] K. C. SivakumaR, Range and group monotonicity of operators, Indian J. Pure Appl. Math., 32, 1 (2001), 85-89.

[31] K. C. Sivakumar, Applications of nonnegative operators to a class of optimization problems, Banach Center Pub., 79 (2008), 197-202.

[32] K. C. SivakUmar, A new characterization of nonnegativity of Moore-Penrose inverses of gram operators, Positivity, 13, 1 (2009), 277-286.

[33] B. S. TAM, Generalized inverses of cone preserving maps, Linear Algebra Appl., 40 (1981), 189-202.

[34] B. S. TAM, A geometric treatment of generalized inverses and semigroups of nonnegative matrices, Linear Algebra. Appl., 41 (1981), 225-272.

[35] L. B. Thomas, Rank factorization of nonnegative matrices, Problems and Solutions, 73-14 (Solution), SIAM Rev., 16, 3 July (1974), 393-394.

[36] J. R. WALL, Rank factorizations of positive operators, Linear Multilinear Algebra, 8, 2 (1979/1980), $137-144$.

[37] J. R. WALl AND E. HAYNSWORTH, Group inverses of certain nonnegative matrices, Linear Algebra Appl., 25 (1979), 271-288.

[38] J. R. WALl AND E. HAYNSworth, Group inverses of certain positive operators, Linear Algebra Appl., 40 (1981), 143-159. 